\title{
Using the Phase-Field Crystal Method in the Multi-Scale Modeling of Microstructure Evolution
}

\author{
N. Provatas, J.A. Dantzig, B. Athreya, P. Chan, P. Stefanovic, N. Goldenfeld, and K.R. Elder
}

The phase-field-crystal method is a new modeling technique that incorporates the periodic nature of a crystal lattice by considering a free energy functional that is minimized by periodic density fields. This simple approach naturally incorporates elastic and plastic deformations and multiple crystal orientations and can be used to study a host of important material processing phenomena, including grain growth, dendritic and eutectic solidification, and epitaxial growth. This paper reviews the phase-field-crystalformalism and its use in modeling of microstructure evolution in pure and binary alloy systems.

\section{INTRODUCTION}

Many novel applications in engineering require improved strength and performance from metal alloys. This is increasingly true in the automotive and aerospace industries where rising fuel costs place a premium on improved strength-to-weight ratios. The most significant new tools for alloy develop- ment exploit microstructure patterning at the nanoscale, where atomic effects are dominant. However, there is still a gap in our understanding of how elasticity, plasticity, grain boundary interactions, and atomic attachment kinetics control microstructure and phase selection during solidification and solid-state transformations.

The "reverse Hall-Petch" effect provides an example of the changes in mechanical behavior at very small length scales. In nanocrystalline materials, the
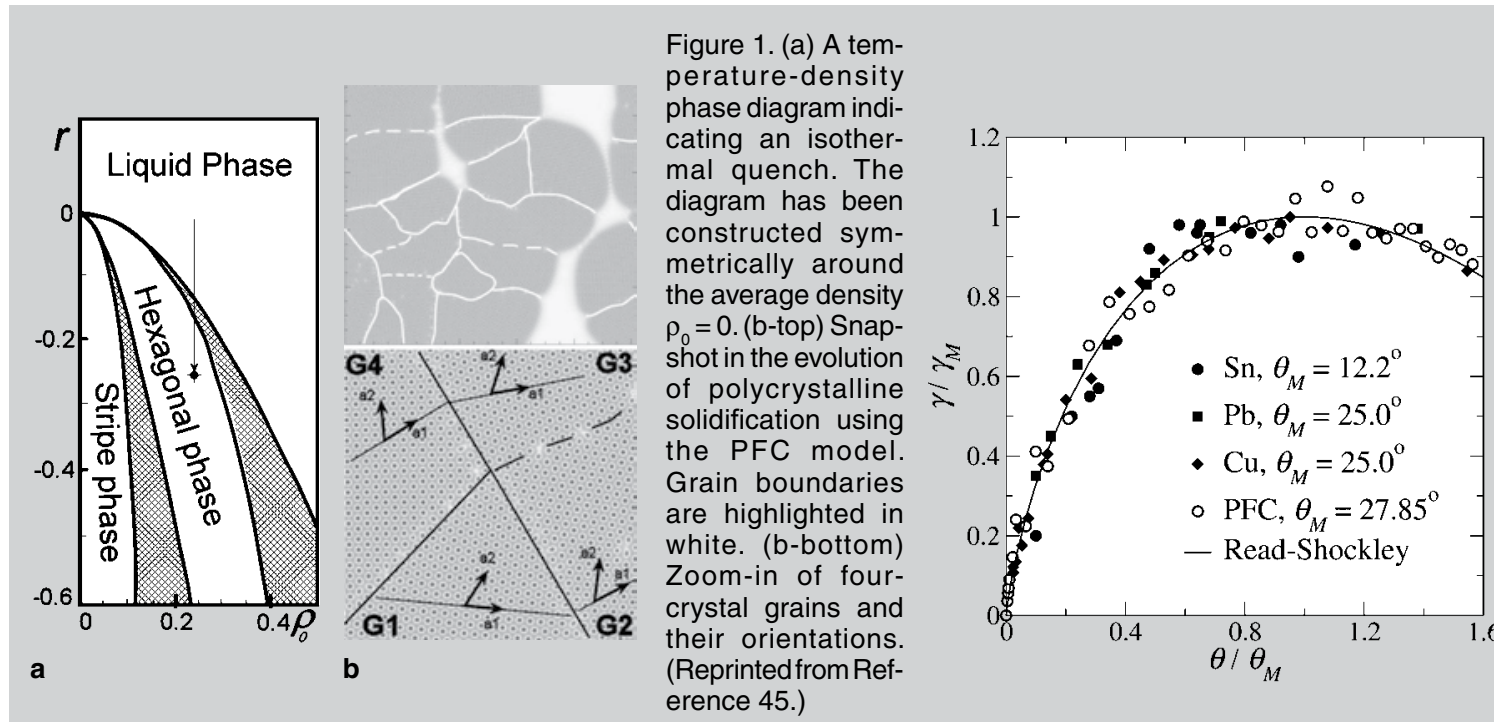

Figure 2. A comparison of the grain boundary energy $(\gamma)$ vs. grain boundary mismatch angle $(\theta)$ from PFC simulations and experiments on tin, ${ }^{51}$ lead, ${ }^{51}$ and copper. ${ }^{22}$ The quantities $\gamma_{m}$ and $\theta_{m}$ represent the values of $\gamma$ and $\theta$ at which the $\gamma$ is a maximum. The line represents the Read-Shockley result. ${ }^{50}$ (Reprinted from Reference 42.)
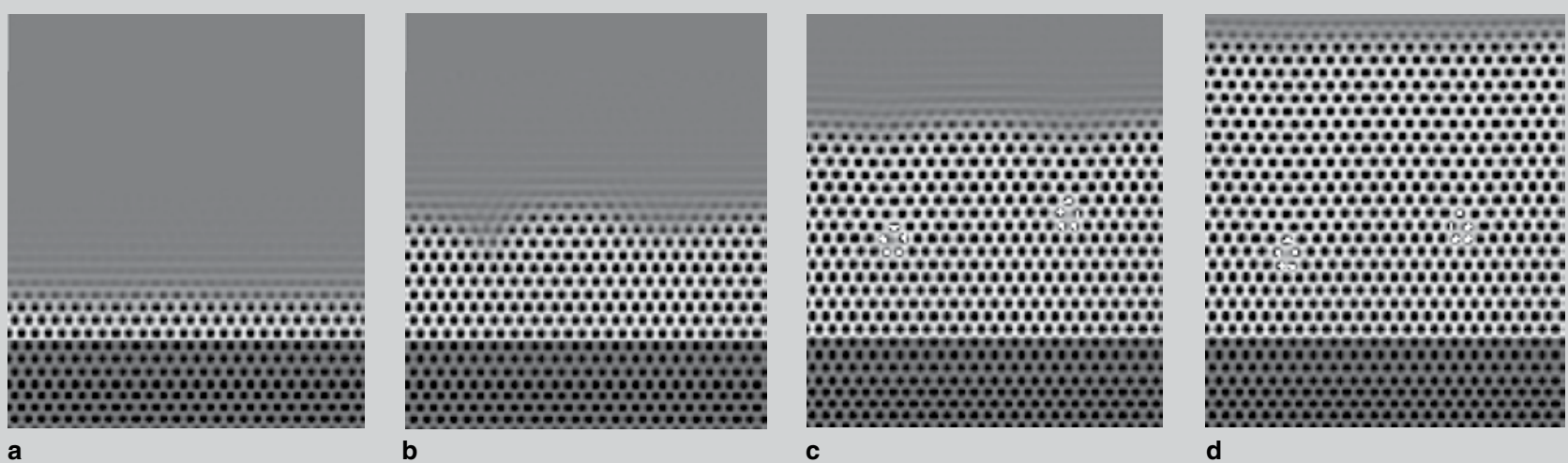

Figure 3. A small portion of a simulation of liquid phase epitaxial growth. (a), (b), (c), and (d) correspond to dimensionless times 150,300 450 , and 600 , respectively. The substrate is highlighted by the darker color and lattice sites near dislocations are marked by small white dots. (Reprinted from Reference 42.) 

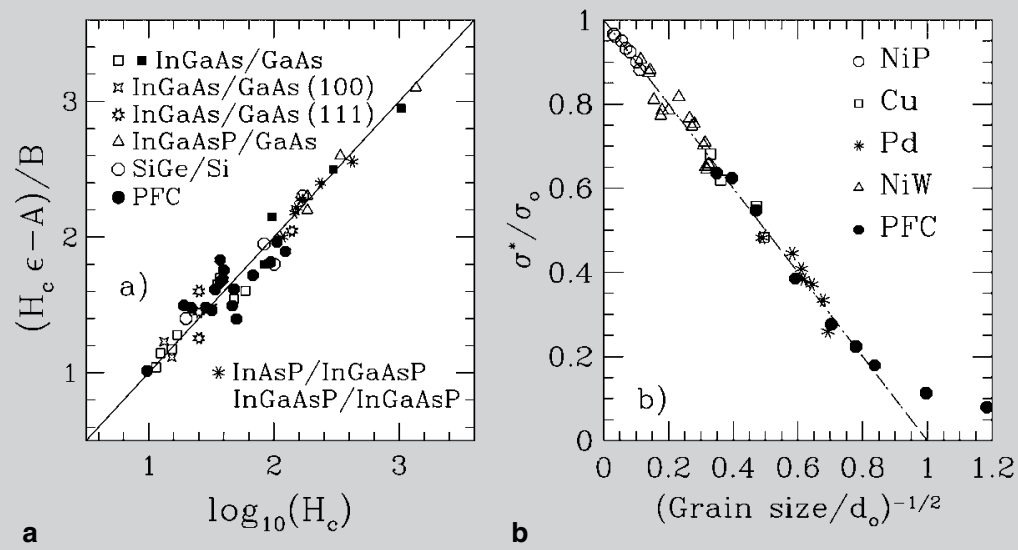

Figure 4. (a) Critical height, $H_{c}$, at which a strained epitaxial film nucleates dislocations for various film/substrate mismatches. The solid square, empty triangle, and circle are data taken from Bolkhovityanov et al., ${ }^{59}$ the data for the empty squares are from Rockett and Kiely; ${ }^{60}$ the empty stars (eight- and four-sided) are from Anan et al. ${ }^{61}$ and the star is from Ogasawara et al. ${ }^{62}(\mathrm{~b})$ Yield stress $\left(\sigma^{*}\right)$ of a polycrystalline solid as a function of grain size. The experimental data are from NiP, ${ }^{63} \mathrm{Cu},{ }^{64-66} \mathrm{Pd},{ }^{64}$ and NiW. ${ }^{67}$ (Reprinted from Reference 42.)
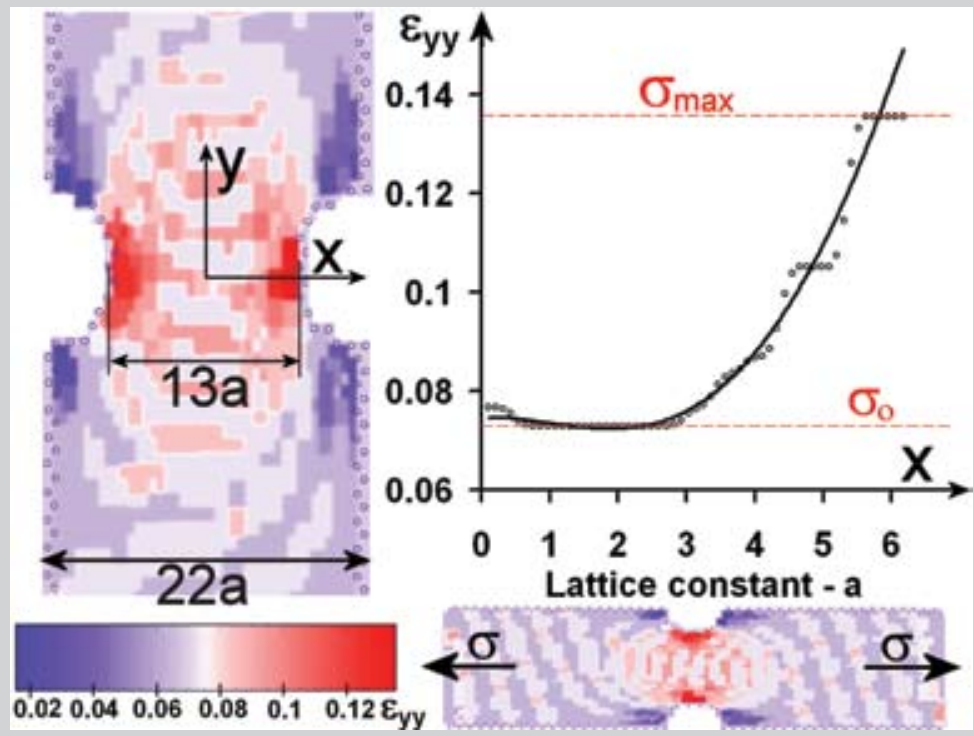

a

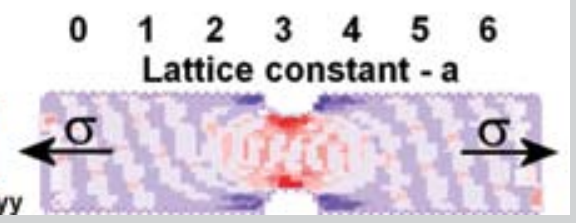

b

Figure 5. Strain concentration in a double-notched sample under a uniaxial tension. (a) A strain map of the center portion of the sample displayed at the bottom. Boundary atoms are highlighted in black. (b) The plot represents a strain profile from the center of the sample into the root of the notch. (Reprinted from Reference 45.)

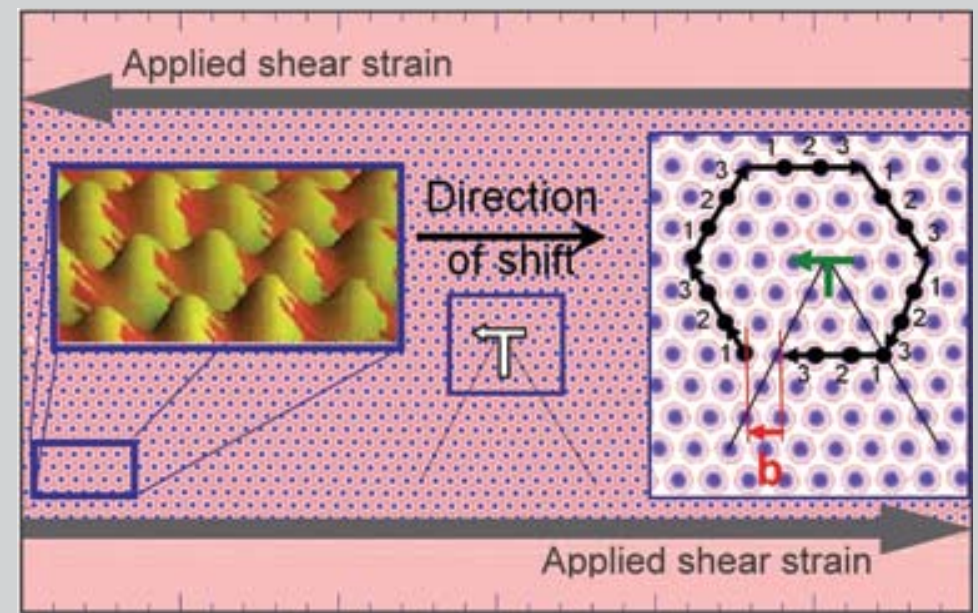

Figure 6. A portion of the sample used to examine dislocation glide velocity. The sample is made by equilibrating a portion of solid-patterned into a rectangular sample-with its coexisting liquid. (Reprinted from Reference 45.) build-up of dislocation networks near grain boundaries is precluded by the small size of the grains, leading to a softening of the material as the grain size decreases, rather than the increase typically seen in larger-grained materials. Another example of nanoscale behavior influencing material properties arises when coherency strains at atomic interfaces control non-equilibrium particle precipitation, which is relevant to solid solution hardening of advanced alloys.

Coherency strains also play a critical role in establishing the stability and structure of epitaxially grown thin films, wherein a few atomic layers of one semiconductor are deposited onto another. Large strains that emerge due to atomic mismatch at the film/substrate interface can lead to buckling, roughening, and ultimately to incoherent growth when dislocations nucleate. The stability and control of such epitaxially grown thin films is key to semiconductor manufacturing.

In these examples and a plethora of others, microstructure formation and material properties derive from an intimate interplay between phase transformation kinetics and elastic and plastic deformations emerging directly from the atomic scale. Modeling these processes thus requires a formalism that simultaneously addresses both the angstrom and micrometer length scales. More daunting still is the need for such a formalism to self-consistently cross the disparate time scales from atomic vibrations to heat and mass transfer that control most phase transformations. This article describes a recently developed approach that meets these requirements, the phase-field-crystal (PFC) model.

\section{PREDICTIVE MODELING OF MICROSTRUCTURE EVOLUTION}

\section{Phase-Field Models}

Computational modeling of microstructure evolution in materials engineering has seen rapid growth in recent years through the use of phase-field methodology. This formalism simulates the dynamics of various fields that characterize the microstructure (e.g., impurity concentration, temperature, crystalline order, and orientation). Among the most extensively studied phenomena using 
the phase-field approach are dendritic growth in pure and binary alloys, ${ }^{1-6}$ spinodal decomposition, ${ }^{7,8}$ order-disorder transition kinetics, ${ }^{9-11}$ and precipitation growth. ${ }^{12}$ In all these phenomena, the dynamics of the appropriate field(s) are assumed to be driven by dissipative minimization of a phenomenological free energy functional.

There have been many significant advances in phase-field modeling in materials science over the last decade. Arguably the most important has been the development of asymptotic analysis techniques that connect the phase-field equations with sharp interface models of solidification in the computationally tractable ("thin interface") limits. ${ }^{13-17}$ These methods have been instrumental in making the phase-field method a viable tool for quantitative modeling of microstructural evolution, especially when coupled with adaptive mesh refinement algorithms, ${ }^{18,19}$ opening up a new window to truly multiscale computation of microstructure evolution. ${ }^{20-24}$

A limitation of traditional phase-field models is that they are formulated in terms of fields that are spatially uniform in equilibrium. This precludes most physical phenomena that arise from the periodic symmetries inherent in crystalline phases, including elastic and plastic deformation, anisotropy, and multiple grain orientations. One way around this problem has been to couple the traditional fields with one or more auxiliary fields that describe, for example, the density of dislocation, ${ }^{25-29}$ the continuum stress and strain fields, ${ }^{30-32}$ and the crystal grain orientation. ${ }^{33-37}$ These approaches have proven quite useful in various applications such as polycrystalline solidification. ${ }^{28,33-40}$ Nevertheless, it has proven quite challenging to incorporate elastoplasticity, diffusive phase transformation kinetics, and anisotropic surface energy effects into a single, thermodynamically consistent model.

\section{The Phase-Field-Crystal Method}

Very recently, the PFC method emerged as a new extension to the phasefield formalism. ${ }^{41-45}$ The PFC formalism abandons the meso-scale order parameter of the traditional phase-field approach in favor of a temporally coarsegrained atomic probability density (APD), or number density. The free

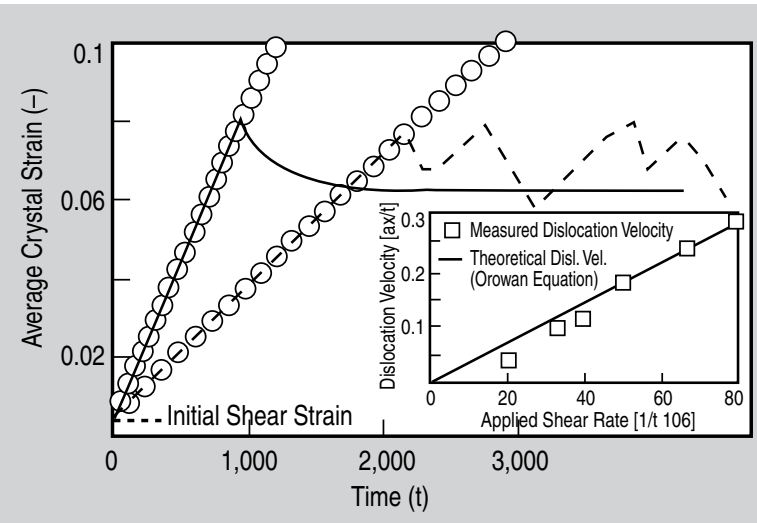

Figure 7. Two regimes of dislocation glide. For high strain rates, continuous glide is observed, while at lower strain rate the dislocation set into a stick-slip motion. Inset: Dislocation glide velocity vs. applied strain rate. (Reprinted from Reference 45.)

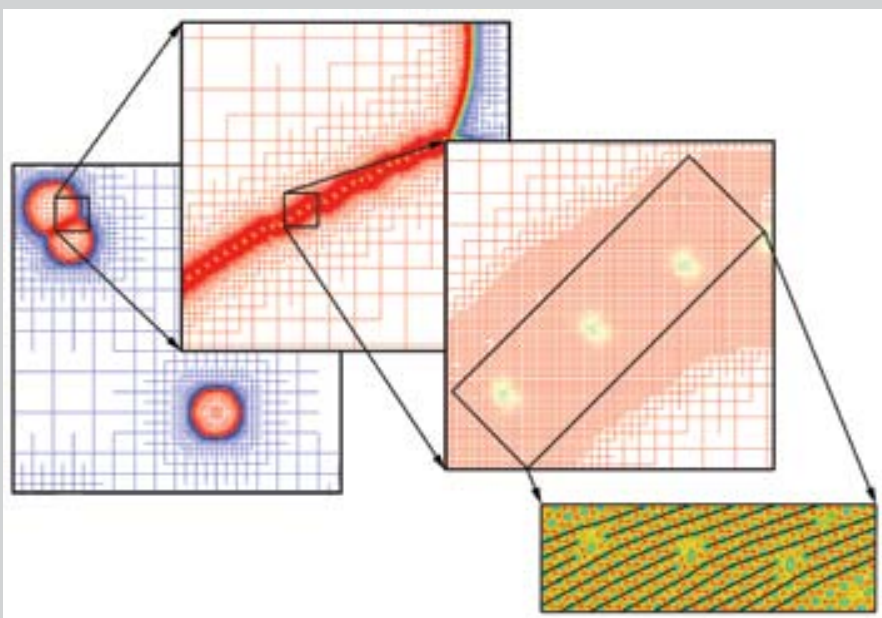

Figure 8. A snapshot from the simulation of solidification of grains in a large domain, 722 $\mathrm{nm}$ square. The series of enlargements shows an approximately $1 \mathrm{~nm} \times 3 \mathrm{~nm}$ segment of the grain boundary formed by the intersection of the growing grains.

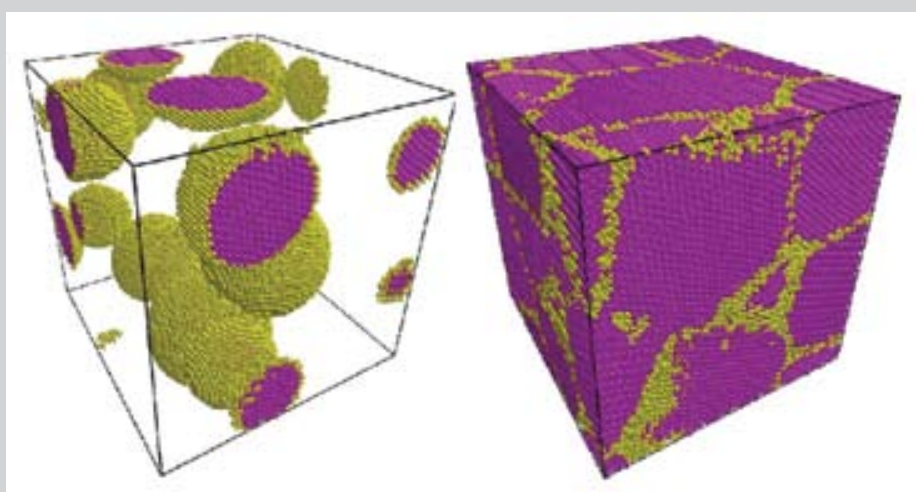

Figure 9. A snapshot of the nucleation and growth of a bcc polycrystal from a supercooled liquid in a system with periodic boundary conditions. In this figure purple corresponds to atomic positions with eight neighbors and yellow to lattice positions at surfaces or dislocations.

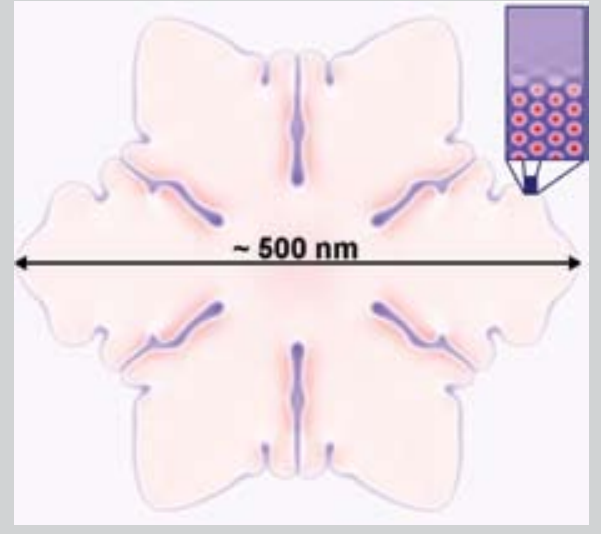

Figure 10. The growth of a two-dimensional hexagonally symmetric solutal dendrite from a supercooled melt. The supersaturation was $\Omega=0.7$ and the average concentration Co was in the hyper-eutectic range. The color map represents the impurity concentration field $\mathrm{c}(\overrightarrow{\mathrm{x}}, \mathrm{t})$, with warm colors representing high composition and blue, low. The inset shows the atomic probability density across the interface near a dendrite tip. A detailed description of the phase diagram for this material is given in Reference 49. 
energy functional of a thermodynamic system, which is constructed in terms of the APD, is minimized by periodic atomic density states for solid phases and a constant density for a liquid. Periodic density states naturally gives rise to elasticity, multiple crystal orientations, and the nucleation and motion of dislocations. Diffusive dynamics of the atomic density field evolve according to the usual dissipative dynamics driven by free energy minimization.

The quintessential advantage of the PFC method is that it integrates atomic vibrations on time scales many orders of magnitude longer than those associated with the Debye frequency $1 / \omega \mathrm{D}$ $10^{-13} \mathrm{~s}$. This allows elastic and plastic effects emerging at the atomic scale to be self-consistently incorporated on the diffusive time scales that govern phasetransformation kinetics. The study of such phenomena using molecular dynamics (MD) simulations is prohibitively expensive since MD is constrained to evolve at the scale of femtoseconds, precluding the study of physics of nanostructured materials, where the relevant length scales are atomic and time scales are mesoscopic.

Recent innovations in the phase-field crystal methodology have further advanced its applicability as a viable tool for multiscale modeling materials properties emergent from the nanoscale. These include a recent extension of original the PFC dynamics to include higher-order time derivatives. ${ }^{45}$ Motivated by hydrodynamics of solids, ${ }^{45,46}$ this dynamical formulation makes it possible to simulate "instantaneous" elastic relaxation, an extension that is important for simulating complex stress propagation and externally imposed strains. ${ }^{45}$

Another innovation has seen the decomposition of the PFC equations of motion into a new set of phase and amplitude equations ${ }^{47,48}$ that are amenable to adaptive mesh refinement schemes. This extension to the PFC formalism has the potential to enable simulations of mesoscopic phenomena $(\mu \mathrm{m} \rightarrow \mathrm{mm})$ that are resolved down to the atomic scale and still incorporate all the physics discussed previously.

More recently still, the PFC formalism for pure materials was extended to include alloys, in which the dynamics of multiple diffusing atomic species can be considered. ${ }^{49}$ This work also showed the formal connection between the PFC methodology and classical density functional theory. Working in conjunction with MD simulations, this connection makes it possible to construct PFC dynamics for various crystal symmetries and materials.

This article presents a brief survey of recent simulations using the PFC method. The aim is to demonstrate how this new modeling paradigm and its recent extensions can be used to address a wide variety of phenomena in materials science occurring across multiple length and time scales, the most important of which emerge at the nanoscale and propagate up to the micro-and macroscale. All the technical details of the methodology and implementations can be found in the references provided in the text and figures.

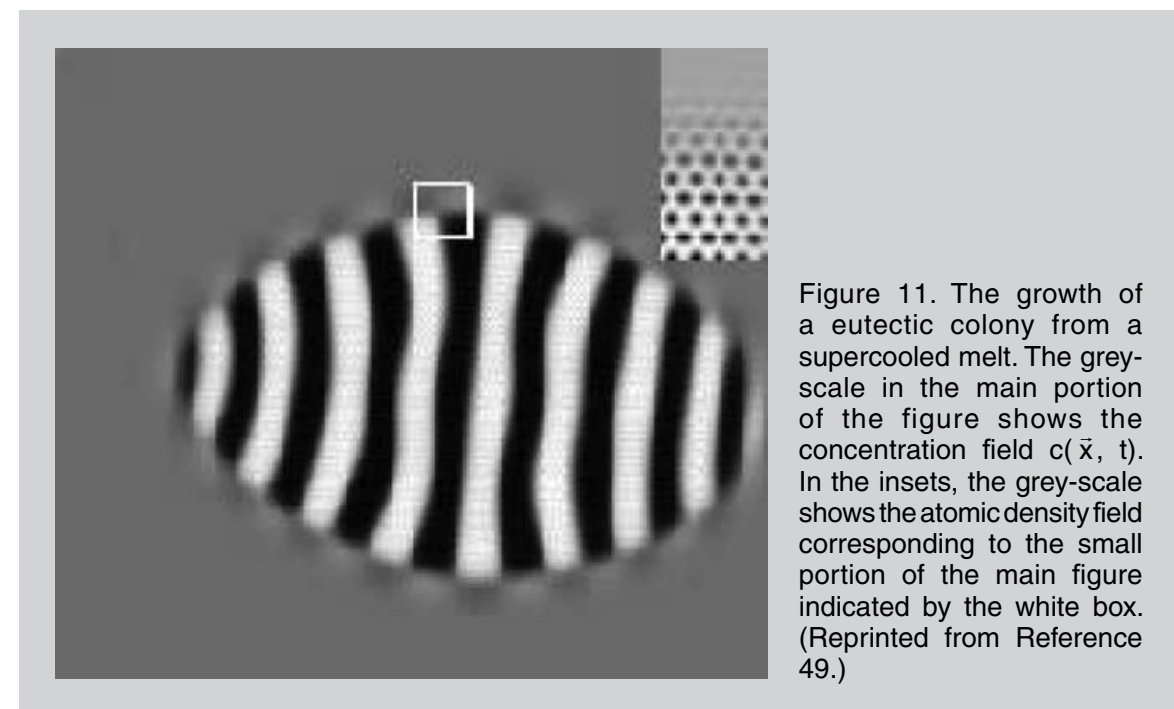

\section{APPLICATIONS OF PHASE- FIELD-CRYSTAL MODELS}

\section{Non-Equilibrium Phenomena in Pure Materials}

Figure 1 shows the phase diagram of the simple free energy corresponding to a pure material. ${ }^{42}$ The y axis (r) corresponds to a scaled temperature and the horizontal axis a scaled average density. The phase diagram contains coexistence between one liquid and two periodic density phases, one of which is a twodimensional hexagonal-close-packed (HCP) phase. A quench from liquid into the HCP phase gives rise to nucleation and growth of nanocrystalline grains of multiple orientations, which impinge to form grain boundaries. The measured grain boundary energies per unit length for such a polycrystalline sample are consistent with the usual Read-Shockley form. ${ }^{38,41,42,50}$ A comparison of the grain boundary energy from the PFC model, the Read-Shockley equation, and several experiments are shown in Figure 2.

In addition to correctly modeling grain-boundary energy the original PFC model was used to study liquid phase epitaxial growth, the reverse Hall-Petch effect in nanocrystalline materials, grain growth, and fracture. An example simulation of liquid phase epitaxial growth is displayed in Figure 3. This figure shows the initial buckling of the interface as predicted by R.J. Asaro and W.A. Tiller $^{53}$ and M. Grinfeld ${ }^{54-56}$ and the eventual nucleation of dislocations. These simulations were used to study the critical height, $\mathrm{H}_{c}$, at which dislocations nucleate as a function of film/substrate lattice mismatch, $\in{ }^{41,42}$ The results were consistent with many experiments and the functional form proposed by J.W. Matthews and A.E. Blakeslee $e^{57,58}$ (i.e., $\left.\mathrm{H}_{c}=\left(\mathrm{A}+\mathrm{B} \log \left(\mathrm{H}_{c}\right)\right) / \varepsilon\right)$, as shown in Figure $4 \mathrm{a}$.

Figure $4 \mathrm{~b}$ summarizes the results of a study of the yield strength of nanocrystalline material. In the simulations nanocrystalline materials were first generated by heterogenous nucleation. The samples were then pulled until plastic deformations occurred and the yield stress, $\sigma^{*}$ was measured. Consistent with the reverse Hall-Petch effect, the yield stress decreased as the inverse square root of the grain size. 


\section{“Quasi-Phonons” and Rapid Strain Relaxation}

The original PFC formulation does not allow for the appropriate separation of times scales required to emulate "instantaneous" elastic relaxation in a solid sample. An extension of this model (dubbed the modified PFC model (MPFC)) developed in Reference 45 includes the ability to relax strains in topologically complex samples much faster than the time scales required for diffusion, yet still orders of magnitude slower than those of real phonon relaxation times. Indeed, the main idea is to emulate rapid elastic relaxation without having to simulate real phonon time scales, which can be done far more effectively with MD.

The properties of rapid strain relaxation inherent in the MPFC model are demonstrated with a simulation of uniaxial tension on a notched sample, illustrated in Figure 5. Strain in the sample accumulates appropriately near the notches, as expected from linear elasticity theory. In treating the case of a double-notched plate, the stress concentration for this geometry is $\mathrm{K}_{\mathrm{t}}$ $=\sigma_{y y}^{\max } / \sigma_{\mathrm{yy}}^{0}=1.8 .{ }^{68}$ This demonstrates that the MPFC model is capable of capturing continuum-scale behavior, even though it evolves at the atomic scale.

The MPFC model can also be used to examine the dynamics of individual dislocations. Figure 6 shows the top part of the crystal initially containing $\mathrm{N}$ atoms and the bottom part $\mathrm{N}+1$. After the sample equilibrated an edge dislocation formed and a constant shear strain rate was applied. The time-averaged dislocation glide velocity $\bar{v}$ was found to be a linear function of the strain rate $\dot{\gamma}$, consistent with classical dislocation theory, which predicts that $\bar{v}=\dot{\gamma} /\left(\rho_{\mathrm{d}} \mathrm{b}\right)$, where $\rho_{d}$ is the dislocation density and $b$ is the magnitude of the Burger's vector.

Computing the average strain in the crystal of Figure 6 as a function of time for different strain rates reveals two regimes of dislocation glide, as shown in Figure 7. The first is characterized by continuous glide (observed at large $\dot{\gamma}$ ) and the second by a stick-slip gliding of the dislocation at low $\dot{\gamma}$. In both cases the applied plastic strain was relieved by the motion of the dislocation, and the time-averaged strain remained constant.

\section{Reformulation for Efficient Computation}

One of the limitations of the PFC methodology is that it is an inherently atomic-scale model. As such it suffers from the same system size limitations as traditional MD. In order to apply the PFC formalism to larger-scale microstructure problems in materials science, it is essential to develop numerically efficient algorithms that enable multiple length scales to be simulated efficiently.

One such approach has been formulated in References 47 and 48, where it was recognized that most of the important dynamics during microstructure formation evolve on wavelengths much longer than the atomic scale, except those occurring near topological defects. Although these nanoscale phenomena comprise only a small fraction of the total system volume, the problem is not directly amenable to adaptive mesh refinement because the density field is periodic at the atomic scale. In the approach of References 47 and 48, the PFC dynamics for the atomic density are reformulated in terms of the amplitude and phase of the density field, rather than the density field itself, because the former vary on length scales much greater than the atomic spacing except near topological defects. The PFC dynamics of the density field are thus re-expressed as commensurate dynamics for the phase and amplitudes in terms of which the APD is expanded. Exploiting this separation of length scales the phase and amplitude equations can be solved using a new adaptive mesh refinement algorithm, ${ }^{69}$ thus enabling a great increase in the system sizes that can be simulated. After solving for the amplitude and phase, the APD is easily reconstructed. This approach thus provides a type of reversible coarse graining for PFC dynamics.

Figure 8 shows a snapshot in time in the evolution of a few grains in a pure material, simulated using this method. The nominal size of the domain is 722 $\mathrm{nm}$ square. The figure shows the grid, clearly demonstrating the dynamic adaptation to the growing crystals. The series of enlargements of the grain boundary region, leading finally to the reconstructed atomic scale density field in the lower right, illustrate the multiscale nature of the simulation. A portion of the low angle boundary with its geometrically necessary dislocations is reconstructed in the lower right hand corner.

\section{Extension to Three Dimensions}

It is straightforward to cast the PFC model in any dimension simply by changing the form of the operator in the free energy density function that controls spatial gradients (i.e., $\left.\mathrm{G}\left(\nabla^{2}\right)\right){ }^{41,42,49}$ Figure 9 shows a simulation of threedimensional (3-D) body-centered cubic (bcc) crystal grains growing into an undercooled melt. In these simulations the grains were nucleated heterogeneously. As shown in Figure 9, small crystallites grow until they impinge to form a 3-D nanocrystal. This simulation contains approximately 400,000 lattice sites.

\section{Binary Alloys}

\section{Solidification}

The PFC methodology has recently been extended to the study of polycrystalline solidification in alloys. ${ }^{49}$ Traditional phase-field models of solidification are typically unable to self-consistently combine bulk elastic and plastic effects with phase transformation kinetics, multiple crystal orientations, and surface tension anisotropy. Moreover, previous approaches have incorporated certain aspects, such as surface tension anisotropy, phenomenologically. In the PFC formalism, these features arise naturally from classical density functional theory. ${ }^{49}$

Figure 10 shows a simulation of an isothermally solidified solutal dendrite with sixfold hexagonal symmetry. The material corresponds to a binary alloy forming a symmetric eutectic phase diagram. ${ }^{49}$ The inset shows the atomic probability density near one of the dendrite tips. The decay of the density fluctuations into the liquid in the inset of Figure 10 defines the atomic-scale sold-liquid interface. It is expected that the fundamental connection of the density field to interatomic potentialsthrough density functional theory-can also help elucidate the mechanisms of 


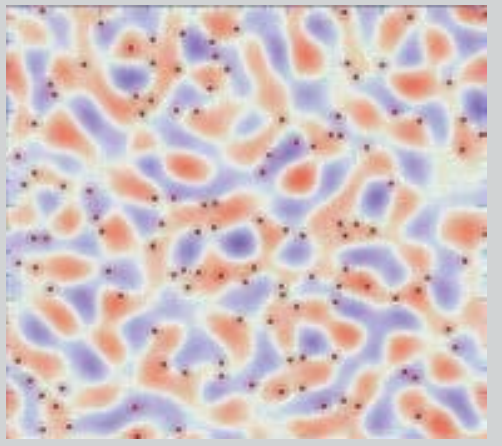

a

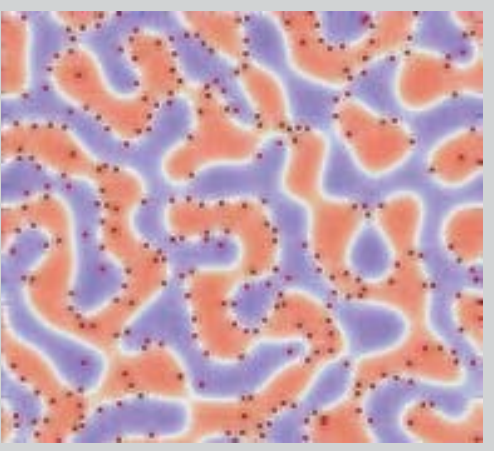

Figure 12. Four time sequences in the evolution of the concentration field (red-blue) superimposed on the corresponding density field (the latter can be resolved by amplifying the on-line version). Dislocations are labeled by a square on the dislocation core surrounded by a circle. The time sequence (a)-(d) corresponds to $t=12,000,24,000,60,000$, and 288,000 (arbitrary units), respectively. The system size is $1,024 \Delta x \times 1,024 \Delta x$, where $\Delta x$ is equal to $1 / 4$ of the lattice constant. The atomic radius (RA) of species $A$ is 25 percent larger than that of species B (RB). (Reprinted from Reference 49.)

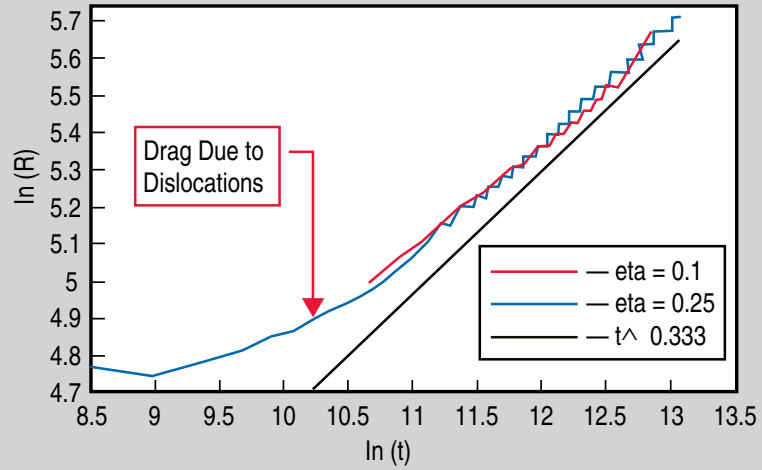

Figure 13. The inverse of the mean wave vector of the (circularly averaged) 2-D structure factor of the concentration field (R) vs. time $(\mathrm{t})$ corresponding to the simulation in Figure 12. Results are shown for two values of the atomic mismatch $\eta=R_{B} / R_{A}$.
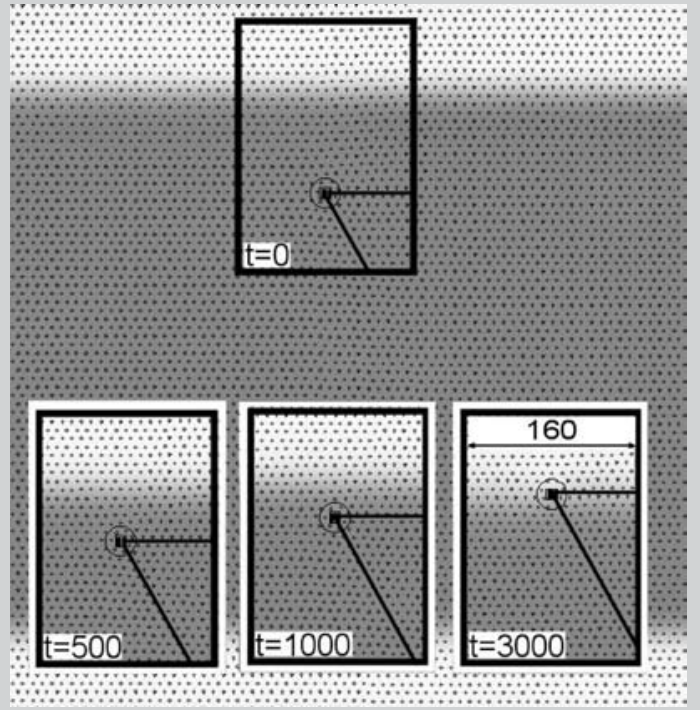

Figure 14. A dislocation migrates toward a coherent phase boundary, thus relaxing mismatch strain. An $800 \times 800$ (units of $\Delta x$ ) portion of the actual simulation domain is shown. The data show four time frames in the motion of the dislocation. Parameters of the simulation are the same as in Figure 12. solute drag and solute trapping, ${ }^{70}$ captured only phenomenologically with traditional phase-field models..$^{71,72}$

Figure 11 illustrates the growth of a eutectic colony from an undercooled melt. The simulation corresponds to the same binary alloy system used to simulate the data of Figure 10. The gray scale corresponds to the two solvus compositions for the chosen undercooling. The inset shows the atomic probability density, which gives rise to coherency strains when the lattice constant is mismatched in the two phases. This will subsequently influence the spacing selection in the microstructure. ${ }^{49}$

\section{Spinodal Decomposition}

Spinodal decomposition has long been used as an important strengthening mechanism in metal alloys. A topic of intense investigation in the experimental and theoretical literature has been the role of dislocations in domain coarsening. It has recently been shown ${ }^{73,74}$ that coherent strains that form at domain boundaries can lead to large strains, which lead to intermediate-time coarsening dynamics slower than the usual $t^{1 / 3}$ growth law. These strains can be relaxed with the flow of mobile dislocations that move toward domain boundaries and lead to an asymptotic speed up of domain coarsening. A scaling analysis of this behavior ${ }^{73}$ has shown these results to be consistent with the sparse experimental data available in the literature. ${ }^{75-78}$

Figure 12 shows the simulation of a temperature quench below the spinodal point of a binary alloy using the alloy PFC model of Reference 49. Simulations began with a homogenous liquid phase at the spinodal concentration. The liquid first solidified into a polycrystalline solid (alpha) phase, which subsequently phase separated as the temperature was lowered below the spinodal. Figure 12 shows the concentration and density fields for four time sequences during the spinodal decomposition process. The dots in the figures denote the locations of dislocation cores. Other parameters for the simulation are given in Reference 49. The spinodal coarsening rate corresponding to the data of Figure 12 exhibits an early and intermediate time regime that is slower that its traditional $\mathrm{t}^{1 / 3}$ behavior, while at late times it asymptotically approaches $t^{1 / 3}$ (Figure 13). 
The simulations in Figure 12 contain both compositional domain boundaries and grain boundaries of different orientations. As a result, the observed dislocation motion is affected by elastic strain energy due to phase separation and curvature driven grain boundary motion. To better isolate the effect of phase separation on dislocations, Figure 14 demonstrates dislocation motion near a coherent interface in the alloy. As in Reference 73, coherent strain energy built up due to compositional differences in the two phases drives the dislocation toward the compositional boundary.

\section{Diffusion Couple}

A recent extension to the PFC model also permits the modeling of persistent vacancies, and differentiation of individual species. Figure 15 shows a zoomin of a snapshot in time from a simulation of diffusion in a periodic array of stripes of two different phases. Notice that diffusion takes place predominantly along grain boundaries in the simulation. By adding noise to the PFC equations, the effect of temperature was also incorporated. By extracting concentration data from the simulation and comparing it with the exact solution for a diffusion couple, the effective diffusion coefficient can be measured. Figure 16 shows a typical result, indicating that the diffusion process in the PFC model is inherently thermally activated when thermal fluctuations are added to the governing equations.

\section{CONCLUSION}

The PFC methodology represents a new paradigm for modeling materials behavior. It provides a continuum-scale representation of the atomic density field that evolves on diffusive time scales. This opens up a wide variety of phenomena for analysis that are not approachable using previous techniques. Specifically, MD simulations are limited to far smaller time scales, while phase-field methods are limited to microscopic length scales and only incorporate atomic-scale parameters effectively (i.e., phenomenologically). Furthermore, most of the hybrid atomic/continuum models have to make limiting assumptions about interactions at the intersection of the two descriptions.

The use of advanced computing algo- rithms will soon lend the PFC methodology to efficient 3-D simulations. This will enable more detailed studies of multiple crystal structures and a more systematic examination of alloy systems. Some of this work has already begun to appear in the literature and at conferences. This new modeling formalism also has great potential to model problems in physical metallurgy, including interaction of multiple dislocations, interactions of dislocations with solute fields, solidification, and a wide variety of other applications. The next few years should bring an exciting array of applications using the PFC method.

\section{ACKNOWLEDGEMENTS}

J. Dantzig, N. Goldenfeld, and P. Chan gratefully acknowledge support from the U.S. National Science Foundation under Grant No. DMR 01-21695. K. Elder would like to acknowledge support from the National Science Foundation under Grant No. DMR-0413062. N. Provatas and $P$. Stefanovic would like to thank the National Sciences and Engineering Research Council of Canadaforfinancial support.

\section{References}

1. J.B. Collins and H. Levine, Phys. Rev. B, 31 (1985), p. 6119.

2.J.S. Langer, Directions in Condensed Matter Physics (Singapore: World Scientific, 1986), p. 164.

3. B. Grossman et al., Phys. Rev. Lett., 71 (1993), p. 3323.

4. K.R. Elder et al., Phys. Rev. Lett., 72 (1994), p. 677. 5. K.R. Elder et al., Phys. Rev. E, 61 (2000), p. 6705.

6. J.A. Warren and W.J. Boettinger, Acta Metall. Mater. A, 43 (1995), p. 689.

7. J.W. Cahn and J.E. Hilliard, J. Chem. Phys., 28 (1958), p. 258.

8. H.E. Cook, Acta Metall., 18 (1970), p. 297.

9. S.M. Allen and J.W. Cahn, Acta Metall., 23 (1975), p. 1017.

10. S.M. Allen and J.W. Cahn, Acta Metall., 24 (1976), p. 425 .

11. S.M. Allen and J.W. Cahn, Acta Metall., 27 (1979), p. 1085.

12. S. Hu and L. Chen, Acta Metall., 49 (2001), p. 463.
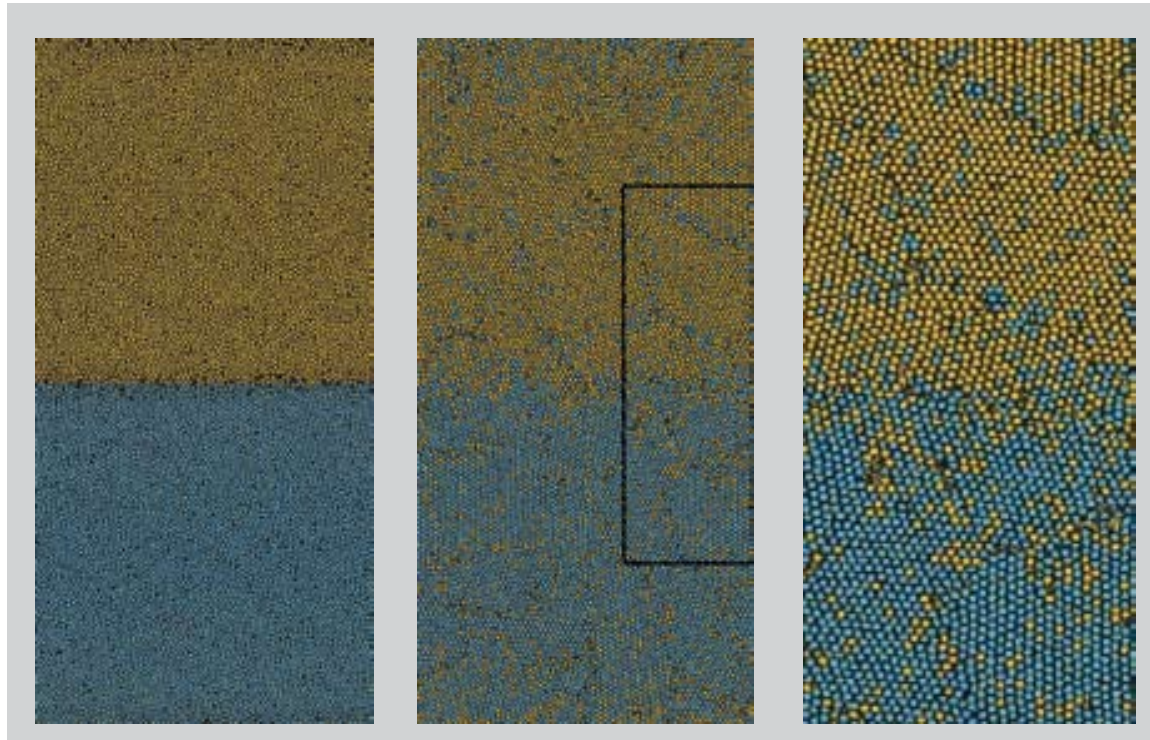

Figure 15. A time sequence from the simulation of diffusion in a binary alloy, showing the densities of $A$ (orange) and $B$ (blue). The initial condition is shown on the left, and all boundaries are periodic. The final panel shows a magnification of the boxed region in the second panel, where one can clearly see the preferential diffusion along grain boundaries and the persistence of vacancies.

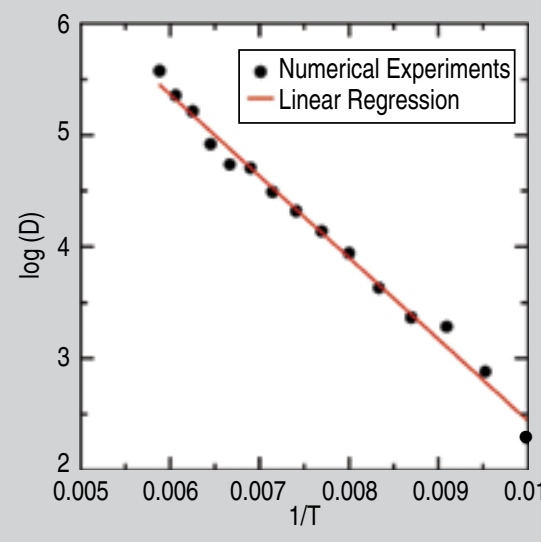

Figure 16. The effective diffusion coefficient obtained by fitting the data of Figure 15 to an exact solution. 
13. A. Karma and W.-J. Rappel, Phys. Rev. E, 53 (1996), p. 3017

14. A. Karma, Phys. Rev. Lett., 87 (2001), p. 115701

15. K.R. Elder et al., Phys. Rev. E, 64 (2001), p. 21604.

16. R. Folch and M. Plapp, Phys. Rev. E, 72 (2005), p. 011602.

17. N. Provatas et al., International Journal of Modern Physics B, 19 (2005), p. 4525.

18. N. Provatas, J. Dantzig, and N. Goldenfeld, Phys. Rev. Lett., 80 (1998), p. 3308

19. N. Provatas, J. Dantzig, and N. Goldenfeld, J. Comp. Phys., 148 (1999), p. 265

20. N. Provatas et al., Phys. Rev. Lett., 82 (1999), p. 4496.

21. N. Provatas et al., Phys. Rev. Lett., 91 (2003), p 155502.

22. C. Lan, Y. Chang, and C. Shih, Acta Mater., 51 (2003), p. 1857

23. M. Greenwood, M. Haataja, and N. Provatas, Phys Rev. Lett., 93 (2004), p. 246101.

24. C. Lan and C. Shih, J. Cryst. Growth, 264 (2004), p. 472.

25. Y.U. Wang et al., Appl. Phys. Lett., vol. 78, 2001, pp. 2324-2326.

26. Y.U. Wang et al., Philos. Mag., 81 (2001), p. 385.

27. Y.U. Wang et al., Acta Mater., volume number? (2001), p. 1847

28. Y.M. Jin and A.G. Khachaturyan, Philos. Mag. Lett., 81 (2001), p. 607

29. M. Haataja et al., Phys. Rev. B, 65 (2002), p 165414

30. L.Q. Chen and A.G. Khachaturyan, Script. Metall. et Mater. 25 (1991), p. 61.

31. Y. Wang and A. Khachaturyan, Acta. Mater., 43 (1995), p. 1837.

32. Y. Wang and A. Khachaturyan, Acta. Mater., 45 (1997), p. 759.

33. L.-Q. Chen and W. Yang, Phys. Rev. B, 50 (1994), p. 15752 .

34. B. Morin et al., Phys. Rev. Lett., 75 (1995), p. 2156.

35. J.A. Warren, R. Kobayashi, and W.C. Carter, J. Cryst. Growth, 211 (2000), p. 18

36. R. Kobayashi, J.A. Warren, and W.C. Carter, Physica D., 140D (2000), p. 141.
37. J.A. Warren, W.C. Carter, and R. Kobayashi, Physica (Amsterdam), 261A (1998), p. 159.

38. J.A. Warren et al., Acta Mater., 51 (2003), p. 6035. 39. L. Granasy et al., Phys. Chem. Glasses, 45 (2004), p. 107

40. L. Granasy, T. Pusztai, and J.A. Warren, J. Phys. Condens. Matter, 16 (2004), p. R1205.

41. K.R. Elder et al., Phys. Rev. Lett., 88 (2002), pp. 245701:1-245701:4.

42. K.R. Elder and M. Grant, Phys. Rev. E, 70 (2004), p. 051605 .

43. J. Berry, M. Grant, and K.R. Elder, Phys. Rev. E, 73 (2006), p. 031609

44. C.V. Achim et al., Phys. Rev. E, 74 (2006), p. 021104.

45. P. Stefanovic, M. Haataja, and N. Provatas, Phys. Rev. Lett., 96 (2006), p. 225504.

46. S. Majaniemi and M. Grant, Phys. Rev. B, 75 (2007), p. 054301.

47. N. Goldenfeld, B.P. Athreya, and J.A. Dantzig, Phys Rev. E, 72 (2005), p. 020601(R).

48. N. Goldenfeld et al., J. Stat. Phys. (to be published in 2007).

49. K. Elder et al., Phys. Rev. B, 75 (2007), p. 064107 50. W.T. Read and W. Shockley, Phys. Rev., 78 (1950), p. 275

51. K. Aust and B. Chalmers, Metal Interfaces (Metals Park, OH: American Society of Metals, 1952), p. 153. 52. N. Gjostein and F. Rhines, Acta Metall., 7 (1959), p. 319

53. R.J. Asaro and W.A. Tiller, Metall. Trans., 3 (1972), p. 1789 .

54. M. Grinfeld, J. Nonlin. Sci., 3 (1993), p. 35.

55. M. Grinfeld, Dokl. Akad. Nauk SSSR, 290 (1986), p. 1358

56. M. Grinfeld, Sov. Phys. Dokl., 31 (1986), p. 831

57. J.W. Matthews and A.E. Blakeslee, J. Cryst. Growth, 27 (1974), p. 118

58. J.W. Matthews, J. Vac. Sci. Technol., 12 (1975), p 126.

59. Y. Bolkhovityanov et al., J. Appl. Phys., 79 (1960), p. 7636.

60. A. Rockett and C. Kiely, Phys. Rev. B, 44 (1991), p. 1154

61. T. Anan, K. Nishi, and S. Sugou, Appl. Phys. Lett., 60 (1992), p. 3159
62. M. Ogasawara et al., J. Appl. Phys., 84 (1998), p. 4775.

63. K. Lu, W. Wei, and J. Wang, Scripta Metall. et Mater. 24 (1990), p. 2319

64. A. Chokshi et al., Scripta Metall., 23 (1989), p 1679

65. J. Schiøtz, F. Di Tolla, and K. Jacobsen, Nature, 391 (1998), p. 561

66. J. Schiøtz et al., Phys. Rev. B, 60 (1999), p. 11971 67. T. Yamasaki et al., Nanostruc. Mater., 10 (1998), p. 375

68. R. Peterson, Stress Concentration Design Factors (New York: Wiley \& Sons., Inc., 1953).

69. B.P. Athreya et al., Phys. Rev. E (submitted 2007).

70. M.J. Aziz and W.J. Boettinger, Acta Metall. Mater., 42 (1994), p. 257

71. N.A. Ahmad et al., Phys. Rev. E, 58 (1998), p. 3436

72. J. Fan, M. Haataja, and N. Provatas, Phys. Rev. E 74 (2006), p. 031602.

73. M. Haataja et al., App. Phys. Lett., 87 (2005), p. 251901.

74. M. Haataja and F. Léonard, Phys. Rev. B, 69 (2004), p. 081201.

75. R.R. Bhat and P.P. Rao, Z. Metallkd., 75 (1994) p. 237.

76. S. Spooner and B. Lefevre, Metall. Trans. A, 11A (1975), p. 1085

77. J. T. Plewes, Metall. Trans. A, 6A (1975), p. 537

78. F. Helmi and L. Zsoldos, Scr. Metall., 11 (1977), p. 899.

N.Provatas and P.Stefanovic are with the Department of Materials Science and Engineering, McMaster University, Hamilton, ON, Canada L8S-4L7; J.A. Dantzig and B. Athreya are with the Department of Mechanical Science and Engineering, University of Illinois at Urbana-Champaign, Urbana, II, 61801; P. Chan and N. Goldenfeld are with the Department of Physics, University of Illinois at Urbana-Champaign, Urbana Illinois, 61801; and K.R. Elder is with the Department of Physics, Oakland University, Rochester, MI, 48309-4487. Prof. Dantzig can be reached at (217) 333-4107; e-mail dantzig@uiuc. edu.

\section{Read JOM On-Line ... Just Like You Do in Print!}
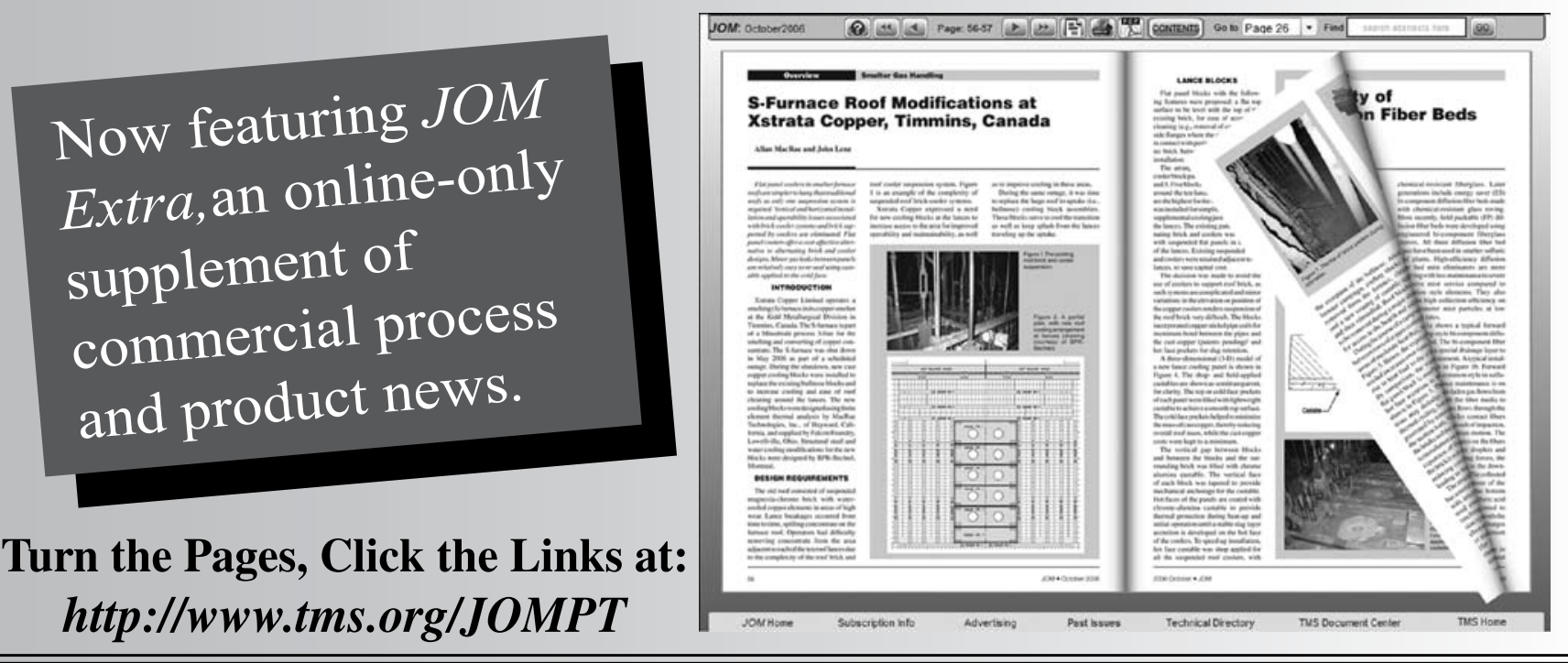